\title{
Resolution des Bundes demokratischer Wissenschaftler gegen die Isolationsfolter in der BRD*
}

Der BdWi wendet sich gegen Isolationsmaßnahmen in Haftanstalten der BRD, die seit Jahren praktiziert werden, in letzter Zeit aber an den sich selbst als politische Gefangene Verstehenden in extremer Form angewendet werden.

Der BdWi fordert, daß Haftpraktiken, die auf die Zerstörung und Brechung von Persönlichkeiten angelegt sind, und die damit in den Zusammenhang von Foltermaßnahmen gehören, da sie lautloser zu den gleichen Resultaten führen, aufgehoben werden.

Der BdWi fühlt sich besonders verpflichtet, die Isolationsfolter anzuprangern, $\mathrm{da}$ es die Wissenschaft selbst ist, die in umfangreichen Forschungen $-\mathrm{z}$. B. an der Hamburger Uni-Klinik - die Zusammenhänge von sozialer Isolation und sensorischer Deprivation untersucht. Für die Anwendung der Ergebnisse bleibr Wissenschaft verantwortlich.

Der BdWi fordert seine Mitglieder auf, öffentlich auf die Abschaffung der Isolationsfolter zu dringen und praktisch die Arbeit der in dieser Sache tätigen Komitees für die Aufklärung über Gefängnisse zu unterstützen.

\section{Praktische Solidarität mit Chile}

Spendenaufruf

Revolutionäres Bewußtsein und Organisation der chilenischen Arbeiterklasse reichen allein nicht aus, um auf Dauer den Angriffen der materiell weit besser ausgerüsteten Reaktion standzuhalten. Chilenische Arbeiter bewachen in diesen Monaten (= chilen. Winter!) unter schwersten Bedingungen Tag und Nacht ihre Betriebe. Sie benötigen dringend einen größeren Posten Schlafsäcke.

In den Industriegürteln, Randsiedlungen und ländlichen Gebieten müssen umgehend Behelfs-Polikliniken errichtet und mit dem nötigsten medizinischen Instrumentarium und Medikamenten ausgestattet werden.

Spendenkonto:

Komitee "Solidarität mit Chile « - Elfriede Kohut

Postscheckkonto 380087 - 108 Berlin-West

Kennwort: Schlafsäcke, Medikamente (KJ)

\footnotetext{
* Diese folgende Resolution wurde auf der Mitgliederversammlung des Bundes demokratischer Wissenschaftler am 8. Juli 1973 in Köln mit großer Mehrheit (bei nur vier Gegenstimmen) verabschiedet.
} 